\title{
Theory and applications of the Vlasov equation ${ }^{\star}$
}

\author{
Francesco Pegoraro ${ }^{1, a}$, Francesco Califano ${ }^{1}$, Giovanni Manfredi ${ }^{2}$, and Philip J. Morrison ${ }^{3}$ \\ 1 Department of Physics "Enrico Fermi", University of Pisa, 56122 Pisa PI, Italy \\ 2 Institut de Physique et Chimie des Matériaux de Strasbourg, CNRS, 67034 Strasbourg, France \\ 3 Physics Department and Institute for Fusion Studies, University of Texas at Austin, Austin, TX78712, USA
}

Received 8 February 2015

Published online 10 March 2015 - C EDP Sciences, Società Italiana di Fisica, Springer-Verlag 2015

\begin{abstract}
Forty articles have been recently published in EPJD as contributions to the topical issue "Theory and Applications of the Vlasov Equation". The aim of this topical issue was to provide a forum for the presentation of a broad variety of scientific results involving the Vlasov equation. In this editorial, after some introductory notes, a brief account is given of the main points addressed in these papers and of the perspectives they open.
\end{abstract}

\section{Introduction}

Referring to the system of equations that now goes under the name of the Vlasov-Poisson system, in his celebrated 1946 paper [1] " On the vibrations of an electronic plasma" Lev Landau wrote a very sharp remark: "these equations were used by A.A. Vlasov for investigation of the vibrations of plasma. However most of his results are incorrect".

Strange indeed is the fate of the name of this fundamental equation for plasma physics that was neither discovered by, nor correctly solved by, the man whose name it bears, although Vlasov correctly recognized [2] that "for a system of charged particles the kinetic equation method which considers only binary interactions - interactions through collisions - is an approximation which is strictly speaking inadequate, so that in the theory of such systems an essential role must be played by the interaction forces, particularly at large distances and, hence, a system of charged particles is, in essence, not a gas but a distinctive system coupled by long-range forces". In fact this equation was used in the context of star dynamics, we would now say of galactic dynamics, one hundred years ago by Jeans [3] and is still generally known in the astrophysics community [4] as the Collisionless Boltzmann equation. This latter denomination however does not do real justice to the full physics content of this equation as, presenting it as a limiting case of the Boltzmann equation, it does not emphasize the novelty introduced by its intrin-

\footnotetext{
* Contribution to the Topical Issue "Theory and Applications of the Vlasov Equation", edited by Francesco Pegoraro, Francesco Califano, Giovanni Manfredi and Philip J. Morrison.

a e-mail: pegoraro@df unipi.it
}

sic nonlinearity that arises from the collective long-range gravitational, or electromagnetic, multibody interactions.

At any rate "the Vlasov equation" is presently the most commonly used trademark of the equation that describes the phase-space classical dynamics (as opposed to quantum dynamics) of multibody systems where the collective (mean field) interaction due to the long-range forces between its elementary constituents overwhelms the effects of their discrete binary interactions, generally known as Coulomb collisions. Depending on the system under consideration the long range interaction may be electromagnetic, in which case the Vlasov equation must be solved together with Maxwell's equations, or gravitational, in which case the Vlasov equation must be solved together with Newton's or Einstein's equations. In all these systems the Vlasov equation allows us to determine the selfconsistent time evolution of the source terms of the electromagnetic or of the gravitational fields. But the solution of the Vlasov equation gives us much more than these source terms as it gives us the time evolution of the full distribution function in phase space of the elementary constituents, particles from now on, of the system. This opens up a new realm of phenomena that are not included in the corresponding set of fluid equations which, at least in principle, would also make it possible to determine a time evolution for the source terms of the gravitational and electromagnetic fields. Of major importance is the phenomenon of phase space mixing of the particle distribution function that is at the basis of the collisionless damping of the perturbations of the particle density and of the electric field discussed by Landau in his 1946 paper. The non dissipative nature of this damping represented a surprising novelty for the plasma community and took some time to become accepted until its experimental verification by Malmberg and Wharton [5] 
nearly twenty years after Landau's original paper. The mathematical subtleties involved in the description of this phenomenon are made evident in the recent work, which was awarded the 2010 Fields medal, by the mathematician C. Villani, see e.g., his recent tutorial presentation in reference [6].

The physics applications of the Vlasov equation range from magnetically confined plasmas for thermonuclear research to space plasmas in planetary magnetospheres and in stellar winds, to relativistic electromagnetic plasmas either produced in the interaction of ultraintense laser pulses with matter or present in the high energy density environment around compact astrophysical objects. Widely different time and spatial scales characterize these systems but they all share the mark of their nonlinear collective behaviour insofar as their macroscopic properties are determined by the excitations of waves and instabilities in conditions that are generally very far from even approximate local thermodynamic equilibrium. The Vlasov equation applies also to the gravitational dynamics of the stellar component of galaxies, where the stars are the particles of the system. In this case the typical characteristic collective dynamical time scale is approximately of the order of one hundredth of the age of the Universe, so that, in general, galactic dynamicists are less interested in the full long term development of the Vlasov dynamics. This includes phenomena such as the so-called violent relaxation [7] of a collapsing stellar system that also occurs on the dynamical time scale.

When solving the Vlasov equation we must address some of the most advanced problems in the physics of dynamical systems, in the stability of Hamiltonian systems with infinite degrees of freedom [8], in kinetic turbulence and Hamiltonian chaos. Recently, the development of accurate numerical schemes that preserve the symplectic nature of the Vlasov dynamics and the availability of increasingly powerful supercomputers have made it possible to explore solutions of the Vlasov equation well beyond the reach of analytical methods. These developments are making the numerical integration of the Vlasov equation an increasing effective and practical investigation tool even in high dimensionality fully nonlinear plasma regimes, thus limiting our dependence on reduced models, such as e.g., fluid-type equations. Together with the ability to produce such collisionless plasma regimes in the laboratory, these numerical investigation tools have brought to light new physics phenomena, e.g., regarding the mechanisms of acceleration of charged particles to ultra high energies in relativistic plasmas. Such collective mechanisms are not easily described within a reductionist approach in terms of elementary single particle processes and their identification is extremely important for understanding the rich workings of our Universe.

It must finally be mentioned that presently there are active research lines that are extending the scope of the Vlasov equation to new physical regimes in a variety of directions, including quantum and incoherent high frequency radiation effects, see e.g., [9,10], electron-positron pair production [11] up to the semiclassical treatment of
non-Abelian field theories in quark-gluon plasmas [12]. Most of these extensions are motivated by their applicability to high energy astrophysics [13]. However in some of these new developments dissipative type of effects, not present in the original collisionless Vlasov equation, are reintroduced due to high energy processes not present at lower plasma energies.

\section{Topical issue}

Partly on the occasion of a conference ${ }^{1}$ held in Nancy in November 2013, a call was launched to all interested researchers worldwide for papers to be published as part of a topical issue in EPJD. This call for papers on the "Theory and Applications of the Vlasov Equation" attracted a large number of very valuable contributions. The full list of the forty papers published, most of them in 2014 (but not in a single issue of EPJD), as contributions to this topical issue are listed on the EPJD web site ${ }^{2}$.

These papers cover a comprehensive set of the different present-day lines of research in the study of the Vlasov equation. These range from revisiting the foundations of the Vlasov equation, its relationship with the single particle dynamics, its Hamiltonian properties, the preservation of such properties in the reduction of the full Vlasov description in fluid-type models, in Landau fluid or gyrokinetic models and finally in numerical implementations, to the description of new, time independent solutions or to the use of special classes of solutions such as the so-called Water Bag distributions, to the study of coherent nonlinear solutions such as solitons and BernsteinGreene-Kruskal and Keen modes. Results of different gyrokinetic numerical investigations were presented, mostly in connection with the physics of magnetized fusion plasmas. In the context of relativistic laser produced plasmas and particle beam generation the problems of the plasma wake field dynamics, of plasma particle acceleration and of the wavebreak in a thermal plasma were addressed. A major subject tackled in this topical issue is the phenomenology of plasma turbulence and intermittence and in particular of phase space turbulence and its investigation within the Vlasov or the gyrokinetic frameworks for both laboratory and space plasmas, but the most numerous set of articles addresses numerical problems and schemes in the numerical integration of the Vlasov-Poisson or Vlasov-Maxwell systems both for magnetized plasmas and for laser produced relativistic plasmas and particle acceleration. Plasma sheaths and low temperature and dusty plasmas are also discussed, the latter in one article as contaminants of a pure electron plasma. On less traditional aspects, we can mention the role of the Vlasov equation in the study of nuclear collective dynamics, the inclusion of exchange interactions in the Vlasov equation for a spin plasma, a cosmological application of an axionic

\footnotetext{
1 VLASOVIA 2013, http://vlasovia2013.event. univ-lorraine.fr/vlasov_home.php.

2 http://epjd.epj.org/component/toc/? task=topic\&id=274
} 
extension of the Maxwell-Vlasov theory and finally the derivation of a Vlasov equation not for the particles in the plasma but for photons and quasi-particles associated with wave modes leading to the definition of a quasi-particle susceptibility.

\section{Conclusions and perspectives}

Two major points are evident from reading the articles collected in this topical issue.

(1) The level of theoretical and numerical maturity that has been reached by the investigation of the collective dynamics of nearly dissipationless electromagnetic or gravitational multi-particle systems: it is now becoming possible to simulate directly within a well defined mathematical framework and without unrealistic parameter restrictions, fully nonlinear kinetic regimes in $6+1$-dimensions (six dimensions in phase space plus time). This has enormous implications from cosmology to fusion science, also at the purely conceptual level where the use of simplified models can easily end up playing a "conservative" role.

(2) The extended scope that the Vlasov equation can have in fields beyond its standard range of applications, including e.g. the novel field of collective Quantum
Electrodynamics in the context of high energy density plasmas.

\section{References}

1. L. Landau, J. Phys. USSR 10, 25 (1946) [J. Exp. Theor. Phys. 16, 574 (1946)]

2. A.A. Vlasov, J. Phys. USSR 9, 25 (1945)

3. J.H. Jeans, Monthly Notices Roy. Astron. Soc. 76, 71 (1915)

4. M. Henon, Astro. Astrophys. 114, 211 (1982)

5. J.H. Malmberg, C.B. Wharton, Phys. Rev. Lett. 13, 184 (1964)

6. C. Villani, Phys. Plasmas 21, 030901 (2014)

7. D. Lynden-Bell, Mon. Notic. Roy. Astron. Soc. 136, 101 (1967)

8. P.J. Morrison, Rev. Mod. Phys. 70, 467 (1998)

9. M. Tamburini, F. Pegoraro, A. Di Piazza, C.H. Keitel, A. Macchi, New J. Phys. 12, 123005 (2010)

10. A. Di Piazza, C. Müller, K.Z. Hatsagortsyan, C.H. Keitel, Rev. Mod. Phys. 84, 1177 (2012)

11. S.S. Bulanov, A.M. Fedotov, F. Pegoraro, J. Exp. Theor. Phys. Lett. 80, 734 (2004)

12. A. Ipp et al., J. Phys.: Conf. Ser. 422, 012028 (2013)

13. S.V. Bulanov, T.Zh. Esirkepov, D. Habs, F. Pegoraro, T. Tajima, Eur. Phys. J. D 55, 483 (2009) 